\title{
Reconstruction of a genome-scale metabolic model for Actinobacillus succinogenes $130 \mathrm{Z}$
}

\author{
Bruno Pereira', Joana Miguel', Paulo Vilaça', Simão Soares', Isabel Rocha ${ }^{2,3}$ and Sónia Carneiro ${ }^{1 *}$ (D)
}

\begin{abstract}
Background: Actinobacillus succinogenes is a promising bacterial catalyst for the bioproduction of succinic acid from low-cost raw materials. In this work, a genome-scale metabolic model was reconstructed and used to assess the metabolic capabilities of this microorganism under producing conditions.

Results: The model, iBP722, was reconstructed based on the functional reannotation of the complete genome sequence of A. succinogenes $130 Z$ and manual inspection of metabolic pathways, covering 1072 enzymatic reactions associated with 722 metabolic genes that involve 713 metabolites. The highly curated model was effective in capturing the growth of A. succinogenes on various carbon sources, as well as the SA production under various growth conditions with fair agreement between experimental and predicted data. Calculated flux distributions under different conditions show that a number of metabolic pathways are affected by the activity of some metabolic enzymes at key nodes in metabolism, including the transport mechanism of carbon sources and the ability to fix carbon dioxide.
\end{abstract}

Conclusions: The established genome-scale metabolic model can be used for model-driven strain design and medium alteration to improve succinic acid yields.

Keywords: Genome-scale metabolic reconstruction, Constraints-based flux analysis, Succinic acid fermentation

\section{Background}

Actinobacillus succinogenes is a gram-negative facultative anaerobic bacterium and is one of the major natural producers of succinic acid (SA). It can grow on a broad range of substrates, including arabinose, cellobiose, fructose, galactose, glucose, lactose, maltose, mannitol, mannose, sucrose and xylose, producing a mixture of by-products (e.g., SA, formic acid (FA), acetic acid (AA), and ethanol (EtOH)) as the main by-products $[1,2]$. Its tolerance to high sugar concentrations (up to 160 g.L $\mathrm{L}^{-1}$ of glucose [3]) and high levels of organic acids [4], as well as its capnophilic nature [5], make this microorganism potentially interesting for the production of SA at the industrial scale. High-titer succinate production using low-cost feedstocks like cane molasses or corn straw has been obtained [2, 6-15]; however, significant

\footnotetext{
* Correspondence: scarneiro@silicolife.com

${ }^{1}$ SilicoLife Lda, Rua do Canastreiro 15, 4715-387 Braga, Portugal

Full list of author information is available at the end of the article
}

amounts of other organic acids were also produced, increasing the downstream processing costs, which makes this bioprocess less competitive. Currently, SA is mainly produced from petrochemical feedstocks through the hydrogenation of maleic acid or maleic anhydride [16]. However, the bio-based production using low $\mathrm{pH}$ yeast fermentation [17-19] or anaerobic fermentation using bacteria [20-23] has been successfully implemented by companies like Myriant [24], BASF [25] or BioAmber [26], offering economically and ecologically attractive alternatives to the conventional petro-based SA production [27-29]. Some examples of SA producing systems are given in Table 1, including naturally-producing bacteria like Basfia succiniciproducens and Mannheimia succiniproducens and genetically engineered organisms such as E. coli or S. cerevisiae. So far, natural producers appear to outperform most engineered strains, but developments in strain design and fermentative processes are expected to promote the production of bio-based SA by metabolically engineered microorganisms. For instance,

(c) The Author(s). 2018 Open Access This article is distributed under the terms of the Creative Commons Attribution 4.0 International License (http://creativecommons.org/licenses/by/4.0/), which permits unrestricted use, distribution, and 
Table 1 Some examples of SA bio-production systems using natural producers or metabolic engineered organisms

\begin{tabular}{|c|c|c|c|c|c|}
\hline Organism & Genetic modifications & Culture conditions & $\begin{array}{l}\text { Carbon } \\
\text { sources (CS) }\end{array}$ & $\begin{array}{l}\text { SA Yield } \\
\left({\left.\mathrm{g} \cdot \mathrm{gcs}^{-1}\right)}\right.\end{array}$ & References \\
\hline B. succiniciproducens & & Anaerobic, continuous & Glycerol & 1.02 & [21] \\
\hline M. succiniproducens & & Anaerobic, batch & Glucose & 0.59 & [71] \\
\hline M. succiniproducens & - Deletion of IdhA, pflB, pta, and ackA genes & Anaerobic, fed-batch & Glucose & 0.76 & {$[30]$} \\
\hline A. succinogenes & & Anaerobic, continuous & Xylose & 0.80 & [63] \\
\hline A. succinogenes & & Anaerobic, batch & Glucose & 0.74 & [72] \\
\hline A. succinogenes & & Anaerobic, repeated fed-batch & Glucose & 0.88 & {$[31]$} \\
\hline E. coli & $\begin{array}{l}\text { - Deletion of pflB, IdhA, ppc genes } \\
\text { - Heterologous expression of pckA } \\
\text { from B. subtilis }\end{array}$ & Anaerobic, batch & Corn stalk hydrolysate & 1.02 & [43] \\
\hline E. coli & $\begin{array}{l}\text { - Deletion of iclR, icd, sdhAB, ackA-pta, poxB } \\
\text { - Heterologous expression of pepc gene } \\
\text { from Sorghum vulgare }\end{array}$ & Aerobic, batch & Glucose & 0.72 & [73] \\
\hline S. cerevisiae & $\begin{array}{l}\text { - Deletion of } s d h 3, \operatorname{ser} 3, \operatorname{ser} 33 \\
\text { - Overexpression of ICL1 }\end{array}$ & Aerobic, batch & Glucose & 0.05 & [37] \\
\hline S. cerevisiae & $\begin{array}{l}\text { - Deletion of pdc1, pdc5, pdc6, fum 1, gpd1 } \\
\text { - Overexpression of pyc2, mdh3, } \\
\text { fumC and frds1 }\end{array}$ & Aerobic, batch & Glucose & 0.14 & [19] \\
\hline
\end{tabular}

Mass yields are given in $\mathrm{g} \cdot \mathrm{g}^{-1}$ of carbon source (CS)

M. succiniproducens has been metabolically engineered by removing competing pathways, resulting in an increase in the SA yield from 0.45 to $0.76 \mathrm{~g}$ of SA per $\mathrm{g}$ of glucose [30].

Under optimized conditions, the A. succinogenes wild-type strain is able to produce up to 98 g.L $\mathrm{L}^{-1}$ of SA with an approximate yield of $90 \%(w / w)$ on glucose [31]. The optimization of bioprocesses has proven to further increase SA production by using high concentrations of carbon dioxide $\left(\mathrm{CO}_{2}\right)$ and/or hydrogen $\left(\mathrm{H}_{2}\right)$ [32, 33]. Other studies have shown that the redox state of the fermentation broth affects SA production, which can be improved by manipulating the supplementation of oxidant and reducing agents $[34,35]$. Yet, due to the accumulation of other fermentative by-products, SA yields are still far below the maximum theoretical yield of 1.12 g.g ${ }^{-1}$ of glucose consumed $\left(\mathrm{Y}_{\mathrm{SA} / \mathrm{Glc}}\right)$ [36]. A comprehensive understanding of the metabolism and the phenotypic responses to environmental perturbations is a major step for developing efficient bioprocesses for SA production.

Genome-scale metabolic models (GSMMs) have proven to be powerful tools for understanding and re-designing the metabolism of microbial strains. For instance, the optimization of SA production in E. coli or $S$. cerevisiae has been achieved by applying metabolic engineering strategies supported by in silico modelling of metabolic networks [37-40]. There are three main pathways for SA biosynthesis, including the tricarboxylic acid (TCA) cycle in the oxidative direction, the glyoxylate shunt and the reductive TCA pathway [41]. Typically, under aerobic conditions, either the oxidative TCA cycle or the glyoxylate shunt can be used for SA production and several studies have used both $S$. cerevisiae and $E$. coli to exploit these metabolic pathways $[38,40]$. The redirection of the carbon flux through the glyoxylate shunt provides some advantages over the oxidative TCA cycle, mainly because the decarboxylation of isocitrate to succinyl-CoA leads to carbon loss [40] and, in the case of $S$. cerevisiae, the SA channelling from the mitochondria to cytosol is avoided as TCA cycle enzymes are located in mitochondria. However, if a reductive TCA pathway is used, a 2-fold maximum theoretical yield (2 mol.mol ${ }^{-1}$ of glucose) can be achieved compared to the oxidative route ( $1 \mathrm{~mol} \cdot \mathrm{mol}^{-1}$ of glucose) [41]. Many organisms, such as E. coli and S. cerevisiae have been tested for SA production under anaerobic conditions using the reductive branch of the TCA cycle [38, 42]. However, reducing power limitations (i.e. $\mathrm{NADH}$ ) or redox balance issues have shown to have an impact on the final SA yields [41]. Metabolic engineering strategies driven by in silico modelling may allow to overcome these disadvantages, both under aerobic and anaerobic conditions. Strategies for increasing energy and/or cofactor pools [43-45], to overcome enzyme limitations [46] or to decrease by-product generation [37] are just a few examples that have been exploited [47].

A. succinogenes produces SA anaerobically through the reductive branch of the TCA cycle (i.e., C4 pathway) using fumarate as the final electron acceptor, which makes this metabolic branch highly dependent on the redox state of cultures. Phosphoenolpyruvate (PEP) node controls the amount of flux that is directed towards the $\mathrm{C} 4$ and $\mathrm{C} 3$ pathways, adjusting the level of fermentative 
products generated by each pathway. Other metabolic nodes like oxaloacetate (OAA) and malate (MAL) have shown to link the $\mathrm{C} 4$ and $\mathrm{C} 3$ pathways via decarboxylating enzymes, i.e. oxaloacetate decarboxylase and the $\mathrm{NADPH}$-dependent malic enzyme, respectively. The split of carbon flux at these branching points is largely influenced by various factors, such as the availability of $\mathrm{CO}_{2}$, $\mathrm{pH}$ or carbon sources. It has been previously shown that an increase in the concentration of dissolved $\mathrm{CO}_{2}$ in the fermentation broth through the supplementation of magnesium carbonate [48] or sodium bicarbonate [32] can promote an increase in the carbon flow toward the C4 pathway, thus increasing the production of SA. The presence of carbonic anhydrases (putative coding gene, Asuc_1199), which interconverts $\mathrm{CO}_{2}$ and bicarbonate $\left(\mathrm{HCO}_{3}{ }^{-}\right)$, may also contribute to increase $\mathrm{CO}_{2}$ fixation $[12,49]$.

Here, a GSMM (named $i$ BP722) representing a wide range of metabolic capabilities of $A$. succinogenes $130 \mathrm{Z}$ is proposed. The model allows predicting and analysing the impact of stoichiometric and physiological constraints known to apply at steady-state conditions. Although the central carbon metabolism of $A$. succinogenes $130 \mathrm{Z}$ has been comprehensively described [32, 33, 50, 51], the overall representation of metabolic pathways associated with various catabolic and anabolic capabilities of the organism are now made available. The biosynthetic pathways for vitamins, cofactors and other biomass building blocks are described, as well as respiratory and energy consuming assimilatory pathway. This model provides a detailed insight on the metabolism of A. succinogenes that can be systematically explored to improve the bioproduction of SA.

\section{Methods}

\section{Metabolic functional annotation}

The complete genome sequence of $A$. succinogenes $130 \mathrm{Z}$ (GenBank accession number NC_009655) [50] was used for the functional annotation of genes based on homology searching methods. The annotated genes with potential metabolic roles were manually inspected and associated with the corresponding coding enzyme(s) and biochemical reaction(s). An internally developed platform was used to compute, assign and curate gene metabolic functions. This platform couples automated annotation tools with manual curation procedures that allows the assignment of metabolic functions to coding sequences (CDSs) of a particular genome. The pipeline consists in five main steps (see Fig. 1):

(1) The application of homology search tools like BLAST [52] and HMMER [53] against sequence databases, such as UniProt [54] to find the best alignment between sequences. A ranked list of hits with the most significant matches to each query is obtained with the respective information, including scores and protein features that may contain Enzyme Commission (EC) numbers.

(2) The computation of "functional scores" based on BLAST and HMMER scores. As the range of enzymatic functions attributed to each CDS can vary from tool to tool, a second score was computed, i.e. so-called functional score, in order to propose the best candidate metabolic functions. Besides BLAST and HMMER scores, a functional score was computed similarly to the method used by Merlin [55], which is based on the frequency of the EC number in the homology hits and on the taxonomy distance between the target strain and other strains within hit results. This weighed functional scores range between 0 and 1 (1 corresponding to a high confidence score).

(3) Assignment of putative metabolic function(s). EC numbers associated with the highest functional score are automatically attributed to each CDS, as well as the corresponding metabolic reaction(s) from an internal reactions database.

(4) The assignment of metabolic functions to each CDS is manually revised, as automatic assignments may fail when more than one high scoring EC number is found and/or EC number(s) are incorrectly associated in databases. Therefore the user is allowed to inspect all putative assignments and select the most appropriate or modify the functional assignment, which can be based on previous knowledge or other assumptions defined by the user. Furthermore, more than one CDS can be associated to the same EC number, which in many cases consists of subunits of the same multimeric enzyme. The classification of multimeric or monomeric subunits is also defined at this stage using an internal database.

(5) After validation of functional assignments and enzyme subunits, the association of metabolic reactions is carried out based on the associated EC number(s) and/or previous knowledge using as a reference an internal reactions database. This step is perhaps the most critical during the reconstruction of the metabolic network, as it will define the set of stoichiometric reactions that characterize a specific organism.

To note that this annotation pipeline is flexible enough, such that unassigned CDSs in step (3) can be later reviewed, particularly during the gap filling process.

\section{Construction of the metabolic network}

The GSMM was initially constructed by compiling the annotated metabolic genes and their corresponding coding 


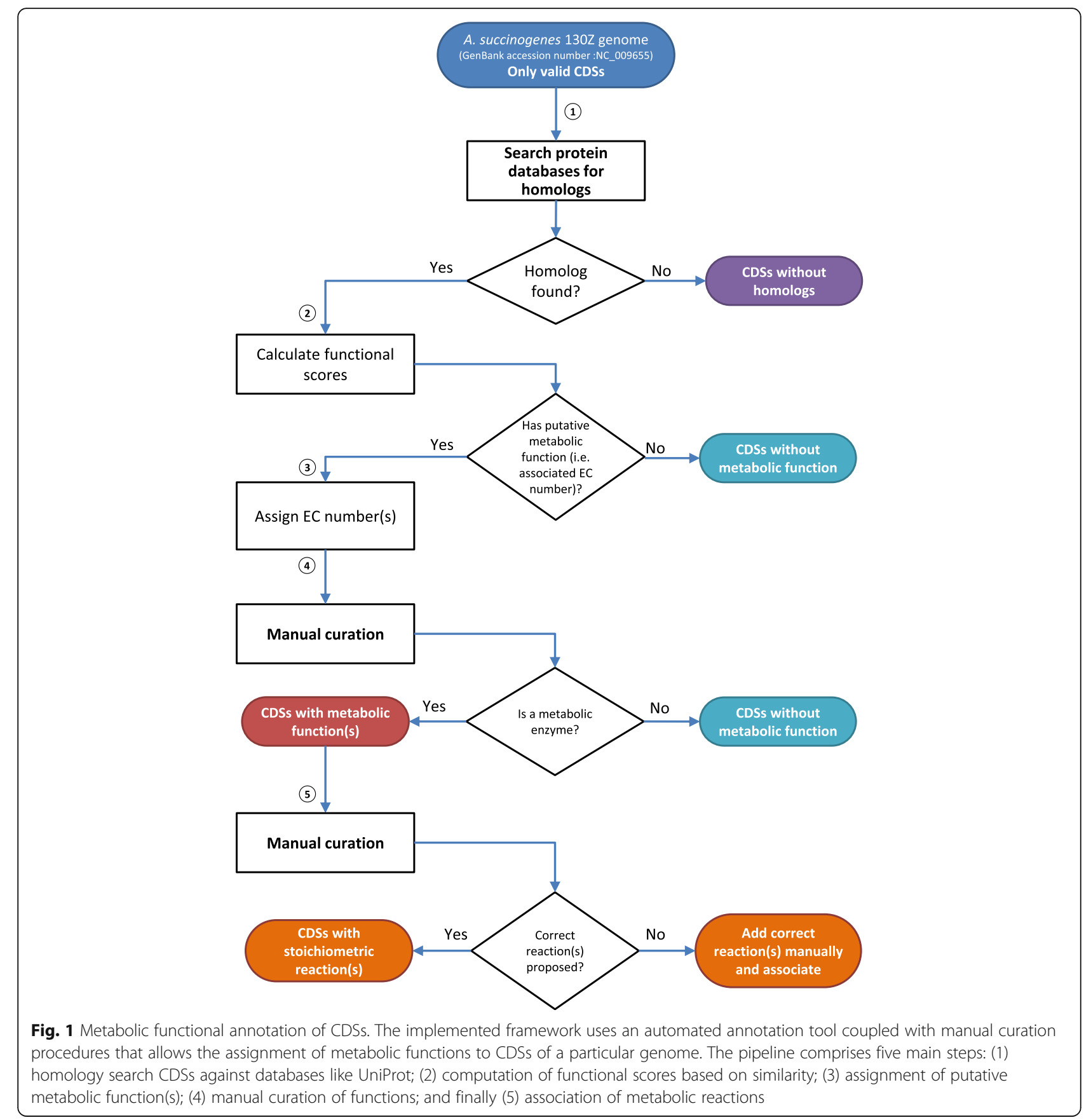

enzyme(s) and biochemical reaction(s). Additionally, spontaneous and transport reactions from databases like KEGG [56] and Transporter Classification Database (TCDB) [57] were added. Some metabolic reactions, although not associated with genes, were also included due to evidences found in literature. The preliminary draft model was then processed to:

- Identify metabolic gaps (or missing reactions) that either consume or produce isolated (or dead-end) metabolites within the metabolic network. Then, each dead-end metabolite was inspected to search for metabolic reactions that consume, produce or transport this metabolite. Typically, MetaCyc or KEGG databases were used for gap-filling, i.e. to identify the sets of biochemical reactions that link each dead-end to a metabolite in the network. When several alternatives are found, a manual inspection is required and sequence-based homology searches using one or more amino acid sequences collected from potential candidates are used to find 
the most likely reactions set in the metabolic network of $A$. succinogenes $130 \mathrm{Z}$.

- Infer and correct the mass and charge balance of biochemical reactions. Stoichiometric coefficients of compounds in reactions are corrected, such that the reaction is balanced for mass and charge, usually by adding missing protons or water molecules.

- Identify and correct the reversibility of reactions based on their thermodynamic properties and information found in literature. Databases like MetaCyc [58] and tools like eQuilibrator [59] were used.

- Include a biomass reaction representing the basic macromolecular composition of $A$. succinogenes in terms of proteins, DNA, RNA, lipopolysaccharide (LPS), phospholipids, peptidoglycan, glycogen and cofactors and vitamins (CAV). The synthesis of each macromolecule was also represented by individual reactions considering the building blocks molar composition. For instance, the synthesis of one gram of protein was calculated based on the average amino acids composition using the set of encoded proteins of the A. succinogenes $130 \mathrm{Z}$ genome, according to the methodology proposed in [60]. The synthesis of other cellular components, like CAV, was calculated assuming that each small molecule is equally present in one gram of CAV. Detailed information on the biomass composition can be found in Additional file 1.

\section{Constraints-based flux analysis}

Basic stoichiometric modelling methods, such as parsimonious flux balance analysis (pFBA) and flux variability analysis (FVA), were used to interrogate the metabolic properties and capabilities of the reconstructed GSMM for A. succinogenes $130 \mathrm{Z}$ under varying environmental conditions. Phenotype simulations were performed by maximizing the biomass reaction assuming growth under defined conditions, i.e. defined minimum media containing basic components required for biomass synthesis, such as vitamins, minerals and trace metals and explicit carbon, nitrogen and sulphur sources. Maximum theoretical product yields were calculated by maximizing the target product instead, ignoring the formation of biomass and ATP maintenance requirements, such that the costs of product biosynthesis in terms of carbon, energy and reducing equivalents were properly evaluated.

\section{Results}

\section{A. succinogenes genome-scale metabolic network}

The construction of the GSMM for A. succinogenes $130 \mathrm{Z}$ was carried out in three different phases: (1) first, metabolic functions were assigned to genes; (2) then, biochemical reactions and enzymatic complexes (assigning proper gene-reaction relationships) were compiled to build a draft metabolic model, (3) which was thereafter completed and corrected by defining a biomass reaction, identifying network gaps and correcting inconsistencies when comparing with reported information.

The final $i$ BP722 model consists of 722 unique genes (open reading frame (ORF) coverage $-35 \%$ ), 1072 reactions and 713 unique metabolites. The model is available as a Systems Biology Markup Language (SBML) file at http://darwin.di.uminho.pt/models and BioModels database [61] assigned with the identifier MODEL1804130001 and detailed information on the curated metabolic network can be found in Additional file 2 .

\section{Model validation}

\section{Predicted growth on different carbon sources}

The $i$ BP722 model was inspected for the ability to simulate the $A$. succinogenes growth on different conditions. Model simulations were performed using the OptFlux software [62] applying FBA-based methods that maximize the biomass reaction under defined conditions, i.e. exchange fluxes were constrained to specific values (usually experimentally measured fluxes) that allow testing growth under defined environmental conditions, such as sole carbon sources. Some exchange fluxes like those associated with the exchange of $\mathrm{CO}_{2}, \mathrm{NH}_{4}{ }^{+}, \mathrm{Pi}, \mathrm{H}^{+}$, vitamins and trace elements were kept unconstrained (i.e. unlimited uptake rates) to provide unlimited basic nutrients for biomass synthesis.

A. succinogenes is auxotrophic for three amino acids: L-glutamate, L-cysteine and L-methionine. Glutamate auxotrophy is due to the organism's inability to synthesize $\alpha$-ketoglutarate, since the genes encoding for isocitrate dehydrogenase and $\alpha$-ketoglutarate oxidoreductase enzymes are absent in the genome. A. succinogenes possesses most of the genes encoding enzymes associated with the cysteine biosynthetic pathway, but the absence of an adenylsulfate kinase to assimilate sulfate prevents the synthesis of hydrogen sulfide required for the synthesis of L-cysteine. This organism also lacks several genes for the biosynthesis of methionine as identified during the functional annotation process. Given that, exchange fluxes associated with amino acid auxotrophies in $A$. succinogenes, such as L-cysteine and L-methionine, were maintained unconstrained, except for L-glutamate that was limited to a minimum flux value to support growth requirements and avoid glutamate consumption as an additional carbon source. Further flux constraints were introduced when inspecting carbon flux distributions in the central carbon metabolism, and are further detailed in Table SI 11 (Additional file 2). For instance, succinyl-CoA ligase reaction was limited to a zero flux to avoid the formation of succinic acid from succinyl-CoA, in order to be consistent with in vivo 
observations [33]. Assuming these flux constraints, growth predictions were computed and compared with in vivo observations $[4,27]$.

Growth phenotypes on 22 different carbon sources under anaerobic conditions were tested and compared with in vivo growth data from [50] (Table 2 and Additional file 3).

The model predicted accurately growth on more than $90 \%$ of the carbon sources, with only two not supporting in silico growth $(\mathrm{FN}=2)$. In silico growth on beta-gentiobiose and D-arabitol was not predicted, as catabolic and transport reactions were not identified in A. succinogenes. Further information on transport activities included in the model is given in Additional file 4.

\section{Predicted yields for fermentation products}

The $i$ BP722 model was further validated by predicting anaerobic production yields and comparing with experimental data from batch and/or chemostat cultures of $A$. succinogenes $130 \mathrm{Z}$ growing on glucose or xylose at different initial concentrations. [32, 51, 63] (Fig. 2). For each condition, carbon uptake rates were defined based on experimental values, except for condition $\mathrm{C}$ that was set to $8 \mathrm{mmol} . \mathrm{gDCW}^{-1} \cdot \mathrm{h}^{-1}$, and then predicted production rates were used to calculate minimum and maximum FVA yields for biomass, SA, AA, FA and $\mathrm{EtOH}$ (Yx/S, YSA/S, YAA/S, YFA/S, YEtOH/S, respectively). FVA spans are given by the difference between the maximum and minimum predicted yields while maintaining $95 \%$ of the maximum biomass formation.

FVA predictions indicate higher yields for $\mathrm{C} 4$ by-products (i.e. SA) compared to $\mathrm{C} 3$ by-products (i.e. $\mathrm{AA}, \mathrm{EtOH}$ and FA), which is in good agreement with experimental data; however predicted mass ratios between fermentative by-products, specifically SA/AA and SA/FA, are higher compared with in vivo observations, especially when considering maximum FVA ratios. Yet, FVA spans indicate a significant flexibility for these ratios, which might explain variations in the in vivo observations.

\section{Improving model predictions}

An FVA analysis was performed to elucidate these discrepancies and the metabolic flexibility associated with

Table 2 Comparison of growth predictions and in vivo tests on 22 carbon sources

\begin{tabular}{llll}
\hline & & In vivo & \\
\hline \multirow{3}{*}{ In silico } & Growth & No growth \\
& Growth & $\mathrm{TP}=19(86 \%)$ & $\mathrm{FP}=0(0 \%)$ \\
& No growth & $\mathrm{FN}=2(9 \%)$ & $\mathrm{TN}=1(5 \%)$
\end{tabular}

True Positives (TP) and True Negatives (TN) indicate the number of carbon sources in which growth phenotypes were correctly predicted, while False Positives (FP) and False Negatives (FN) indicate the number carbon sources in which in silico predictions did not match in vivo observations
C3 and C4 metabolic pathways in A. succinogenes was examined. Given a set of flux constraints (e.g. substrate uptake rates, $\mathrm{q}_{S}$ ), the predicted flux spans for the main reactions in the central carbon metabolism were calculated (Fig. 3a). Flux spans are given by the difference between the maximum and minimum predicted flux values of each metabolic reaction while maintaining $95 \%$ of the maximum biomass formation. As shown in Fig. 3b, the largest flux spans were associated with reactions around the PEP node (e.g. PEP carboxykinase (PPCK) and pyruvate kinase (PYK)), while reactions associated to the Embden-Meyerhoff-Parnas pathway (glucose-6-phosphate isomerase (PGI), phosphofructokinase (PFK) and enolase (ENO)) presented the lowest flux spans. Metabolic flux data estimated from ${ }^{13} \mathrm{C}$-labeling experiments [33] was further used to assess the accuracy of predicted flux spans. Most in vivo measurements were between estimated flux ranges, except for the $\mathrm{CO}_{2}$ exchange and formate dehydrogenase (FDHmq) reactions, indicating that the NADH-producing FDHmq reaction should be active and $\mathrm{CO}_{2}$ exchange flux should be lower. Changes in flux constraints associated with $\mathrm{CO}_{2}$ uptake (from unlimited to a maximum of $4 \mathrm{mmol} . \mathrm{gDCW}^{-1} \cdot \mathrm{h}^{-1}$ ) altered predicted flux spans (Fig. 3c), especially for FDHmq, pyruvate-formate lyase (PFL) and the FA exchange reaction (EX_FA), indicating a higher flexibility in metabolic activities linked to FA accumulation.

The production of SA in A. succinogenes is influenced by several factors, namely the utilized carbon sources [64] or the availability of $\mathrm{CO}_{2}[5,33,48]$. The $i \mathrm{BP} 722$ model was investigated for predicting the metabolic flexibility associated with the production of SA when changing $\mathrm{CO}_{2}$ availability or carbon sources, as well as the reversibility of metabolic reactions like malic enzyme (ME2) (Fig. 4). FVA yields for maximum growth simulations show that the production of reduced by-products, particularly $\mathrm{EtOH}$, changes with the carbon source. As presented in Fig. 4a, the minimum and maximum FVA yields for ALCD2x under D-sorbitol growth conditions (0.62 and $0.69 \mathrm{~mol} \cdot \mathrm{mol}^{-1}$ ) were higher compared to glucose (0.14 and $0.22 \mathrm{~mol}^{\mathrm{mol}} \mathrm{l}^{-1}$, respectively), with the consequent accumulation of higher amounts of EtOH. Under glucose conditions most of the carbon flux through the $\mathrm{C} 3$ branch is redirected toward the production of AA instead, via acetate kinase $(\mathrm{ACKr})$ with the production of ATP (minimum and maximum FVA yields of 0.47 and $0.55 \mathrm{~mol} . \mathrm{mol}^{-1}$, respectively). Interestingly, however, is that predicted FVA yields for SA production hardly change between glucose and D-sorbitol growth conditions when considering the same transport mechanism, i.e. PEP:sugar phosphotransferase system (PEP:PTS). Yet, assuming that both transport mechanisms could be active (i.e., symport and PEP:PTS) for glucose uptake would increase SA minimum and 
Glucose (A1)

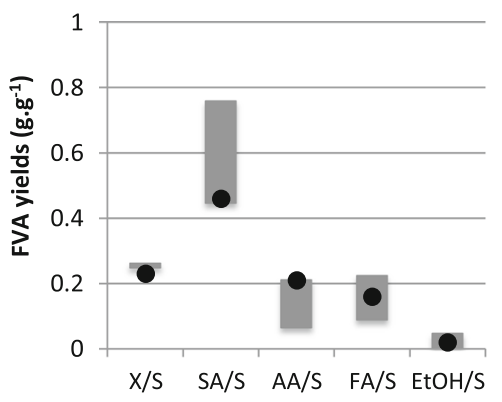

Glucose (B1)

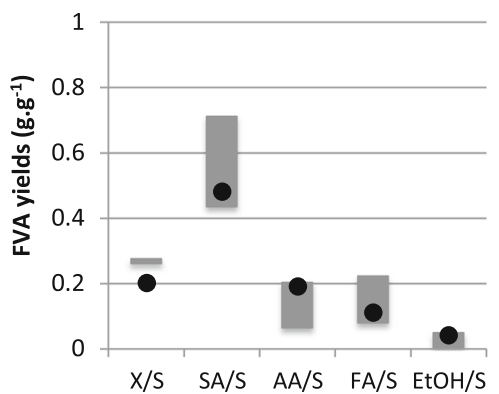

Xylose (C1)

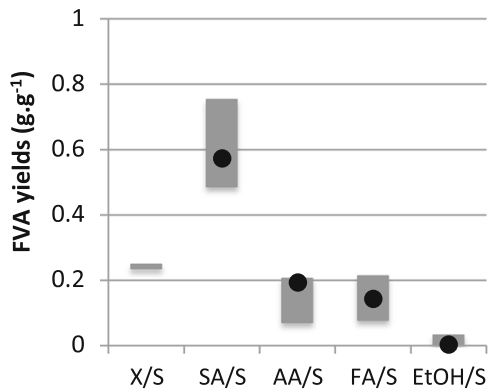

Glucose (A2)
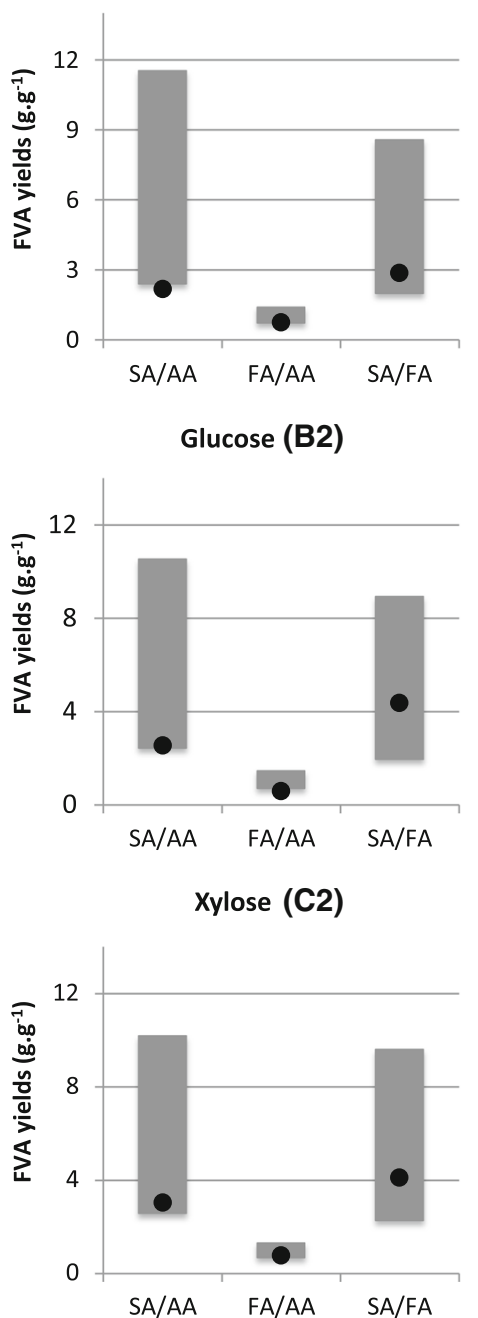

Fig. 2 Experimental values (filled dots) versus predicted FVA yields (floating bars) from A. succinogenes $130 Z$ cultures on glucose (A and B) and xylose (C). Predicted minimum and maximum FVA yields for biomass, SA, AA, FA and EtOH ( $Y_{x / S}, Y_{S A / S}, Y_{A A / S}, Y_{F A / S}, Y_{\text {EtOH/S, }}$, respectively) were estimated while maintaining 95\% of the maximum biomass formation (A1, B1 and C1). Similarly, minimum and maximum FVA yields between fermentative by-products (SA/AA, FA/AA and SA/FA) were estimated while maintaining 95\% of the maximum biomass formation (A2, B2 and C2). In silico predictions were performed by setting the substrate uptake rate $\left(q_{s}\right)$ to the experimental value (except in condition $C$ that was set to $\left.8 \mathrm{mmol} . \mathrm{gDCW} \mathrm{W}^{-1} \cdot \mathrm{h}^{-1}\right)$. Experimental parameters for conditions A and B were obtained from batch cultures with defined medium (AM3) supplemented with $50 \mathrm{mM}$ glucose and $150 \mathrm{mM} \mathrm{NaHCO}_{3}$ under anaerobic conditions [32, 51]; while parameters for C were obtained from a continuous culture at a dilution rate of $0.05 \mathrm{~h}^{-1}$ under anaerobic conditions with supplemented medium $\left(6 \mathrm{~g} \cdot \mathrm{L}^{-1}\right.$ yeast extract, $10 \mathrm{~g} . \mathrm{L}^{-1}$ corn steep liquor and 50-85 g. $\mathrm{L}^{-1}$ xylose). No biomass yield was experimentally determined for this condition [63]. Mass yields are given in $\mathrm{g} . \mathrm{g}^{-1}$

maximum FVA yields from 0.63 and 0.67 to 0.82 and 0.96 mol. $\mathrm{mol}^{-1}$, respectively.

The flux exchange between the $\mathrm{C} 3$ and $\mathrm{C} 4$ branches has been also investigated as a major factor affecting the metabolic flexibility of sugars fermentation in A. succinogenes [33]. The decarboxylation of L-malate to pyruvate (PYR) reducing $\mathrm{NADP}^{+}$to $\mathrm{NADPH}$ by $\mathrm{ME} 2$, may have a major role in the fermentative metabolism. Although the thermodynamics of this reaction is not conclusive regarding its reversibility, the metabolic network was tested using both the forward and the reverse directions.
FVA results (Fig. 4b) show that SA production should increase when ME2 occurs in the reverse direction, especially for $\mathrm{D}$-sorbitol conditions, redirecting most of the PYR pool toward the C4 branch. Consequently, a greater metabolic flexibility in SA production, especially under glucose growth conditions, was predicted.

The availability of $\mathrm{CO}_{2}$ was also shown to influence the production of SA (Fig. 4c). Model predictions indicated that maximum SA yields on glucose can decrease nearly $20 \%$ when decreasing the maximum $\mathrm{CO}_{2}$ uptake rates by $50 \%$. A shift in carbon flux distributions is 

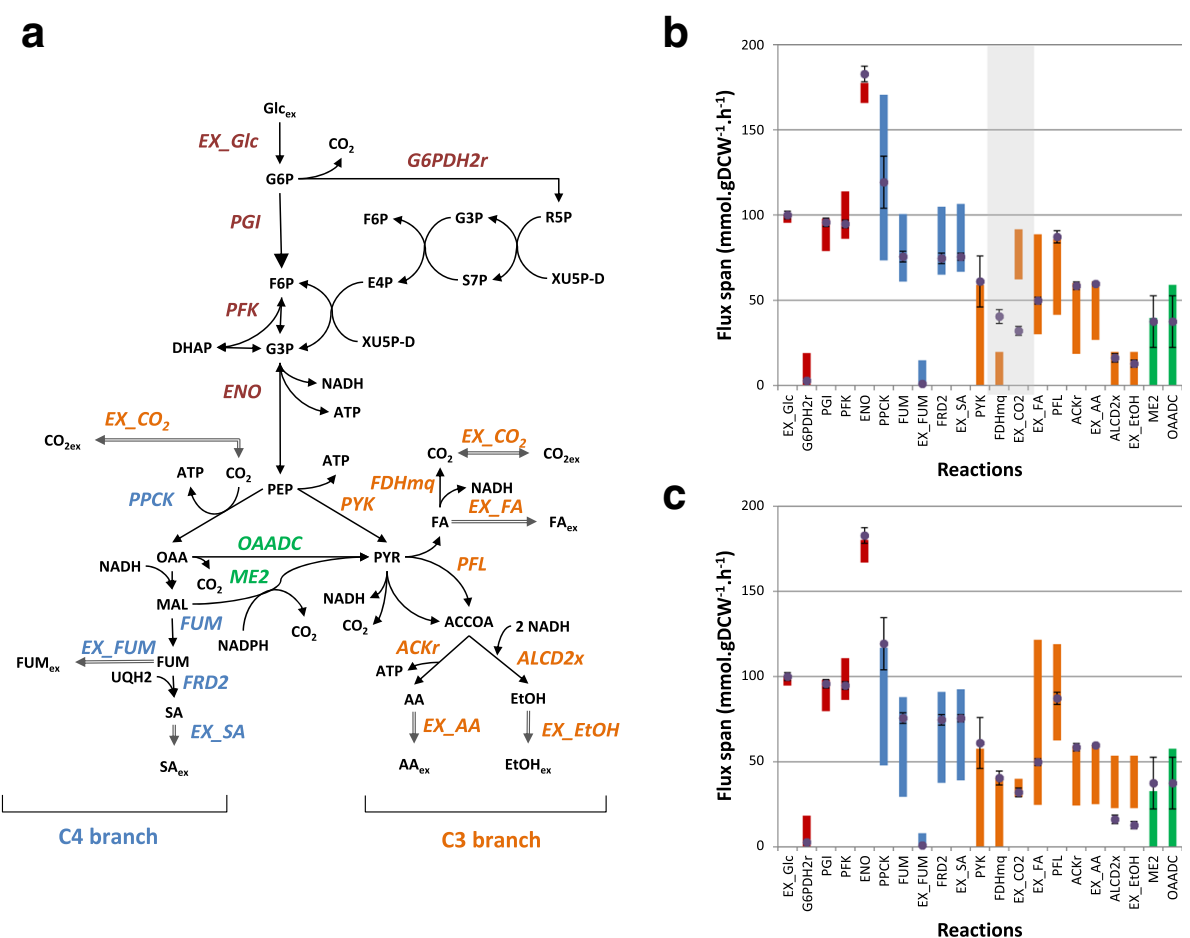

Fig. 3 Metabolic flux spans of the A. succinogenes central carbon metabolism. The FVA analysis covered individual reactions represented in (a). Flux spans (represented by floating coloured bars) define the flux range of individual reactions while maintaining $95 \%$ of the maximum biomass formation (b) and further constraining the maximum uptake rate of $\mathrm{CO}_{2}$ to 4 mmol.gDCW ${ }^{-1} \cdot \mathrm{h}^{-1}$ (c). In vivo flux measurements from ${ }^{13} \mathrm{C}$-labeling experiments [33] (represented by filled dots with error bars) are also depicted.

observed when decreasing $\mathrm{CO}_{2}$ uptake levels, redirecting most of the carbon flux toward the production of $\mathrm{C} 3$ by-products like FA and EtOH.

\section{Discussion}

GSMMs are powerful tools to explore the metabolism of biological systems. In this work, the $i \mathrm{BP} 722$ model of $A$. succinogenes $130 \mathrm{Z}$ was reconstructed and used as a platform for the in silico analysis of the metabolic behaviour of this organism during anaerobic growth. The major end-product is SA, but significant amounts of other by-products such as EtOH, FA and AA are also accumulated. The possibility to predict and adjust the fermentative metabolism of $A$. succinogenes $130 \mathrm{Z}$ under different conditions brings new opportunities to exploit this host as a platform for the industrial production of SA and other reduced by-products.

In silico simulations were carried out using pFBA and FVA methods predicting growth behaviour under chemically defined medium. Model predictions were validated using reported physiological data and flux distributions from ${ }^{13} \mathrm{C}$ experiments found in literature [32, 33, 51]. The $i$ BP722 model supports growth predictions on 19 carbon sources under anaerobic conditions (Additional file 3), including C6 and C5 sugars that were found to enter the cell mainly through active transport systems (Additional file 4). The only two carbon sources with incorrect growth predictions were $\beta$-gentiobiose and $\mathrm{D}$-arabitol, due to the absence of transport and catabolic pathways in the model, since no coding proteins were found. On the other hand, no-growth predictions for glycerol were correctly identified, though transport and catabolic reactions for glycerol consumption are present in the model. According to in vivo growth experiments [65], no cellular growth on glycerol as a sole carbon source is observed under anaerobic conditions, possibly caused by redox imbalance under anaerobiosis. However, the addition of external electron acceptors like dimethylsulfoxide (DMSO) has shown to recover cellular growth [35], which was also confirmed by in silico analysis (Additional file 5).

Further validations included the comparison of in vivo measurements and in silico FVA predictions for minimum and maximum yields under various conditions (Fig. 2). Despite being relatively variable (from 0.20 to 0.23 g.g $\mathrm{g}^{-1}$ under glucose conditions), experimental values for biomass yields were used to validate model predictions. Variations in experimental conditions, particularly associated with culture media that is often supplemented with yeast extract, interfering with carbon 


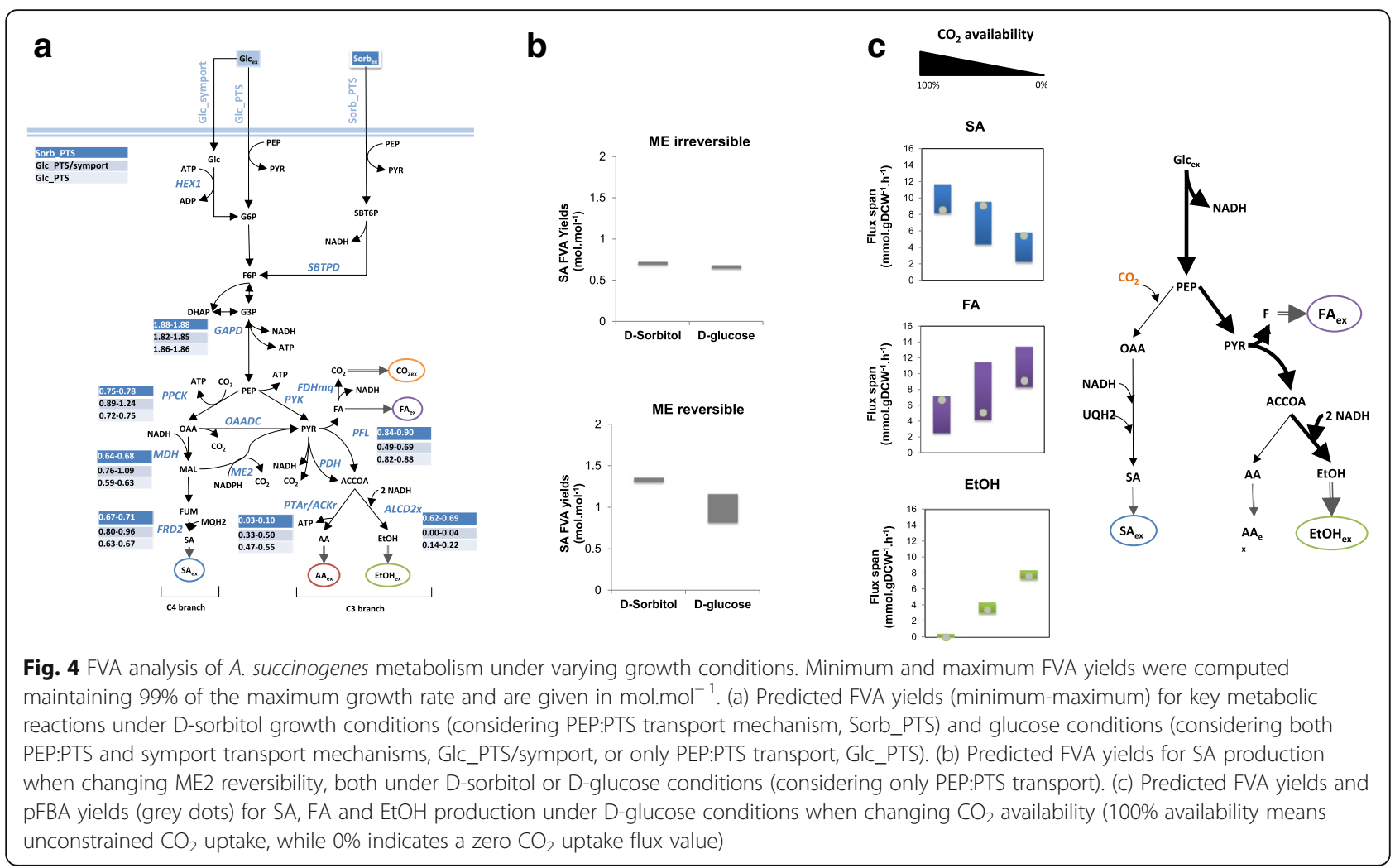

yields, or the initial sugar concentration that may affect bacterial growth due to substrate inhibition [66], may explain these differences. Nevertheless, biomass composition represented in the model is based on the work of McKinlay and co-workers [51], which is expected to provide accurate in silico predictions, especially regarding metabolic requirements to generate biomass contents per unit of substrate (i.e. biomass yields, $\mathrm{Y}_{\mathrm{x} / \mathrm{s}}$ ). Additional reaction constraints were included in the model to improve model predictions. For instance, flux constraints of L-glutamate uptake and succinyl-CoA synthase were changed to improve carbon-to-nitrogen ratios according to experimental measurements [51]. Moreover, the energetic requirement for non-growth associated maintenance, i.e. the amount of ATP spent for cellular maintenance without growth, was adjusted to improve predicted biomass yields.

Fermentative products ratios were also compared, showing some consistency between experimental measurements and FVA predictions. Predicted mass ratios for SA/AA and SA/FA are higher than for SA/FA, which is consistent with in vivo data; but FVA spans for SA/ AA and SA/FA suggest a huge flexibility in these ratios. This suggests that carbon flux distributions in the C3 branch are rather challenging to predict. In fact, FVA flux spans of fermentative pathways (Fig. 3b) indicate a high metabolic flexibility in the accumulation of by-products which, in some cases are inconsistent with experimental measurements. However, when using data from ${ }^{13} \mathrm{C}$-labeling experiments [51] to constrain fluxes, in particular the $\mathrm{CO}_{2}$ uptake rate (Fig. 3c), model predictions improved, especially for reactions associated with FA accumulation (PFL, FDHmq and EX_FA).

Overall, the $i \mathrm{BP} 722$ model allow us to evaluate the production of SA, showing that maximum theoretical yield for SA (1.1 g per $\mathrm{g}$ of glucose, assuming a symport system and no ATP requirements for maintenance) is comparable to those predicted using E. coli or S. cerevisiae models (1.1 and $0.8 \mathrm{~g}$ per $\mathrm{g}$ of glucose for anaerobic conditions using iJO1366 [67] and iMM904 [68], respectively, under the same previous assumptions). Thus, metabolic capabilities seem equivalent to other organisms being exploited for SA production. It also allows describing the impact of growth conditions on the production of $\mathrm{C} 3$ and $\mathrm{C} 4$ fermentative by-products. The carbon split between $\mathrm{C} 4$ and $\mathrm{C} 3$ pathways has been investigated and showed to be influenced by various factors like the available reducing power (i.e. NADH/NAD ${ }^{+}$ ratio) or $\mathrm{CO}_{2}$ availability, therefore affecting the SA production in A. succinogenes growing cultures [64, 69]. In silico predicted SA yields are higher with more reduced carbon sources (e.g. minimum and maximum FVA yields of 0.44 and $0.46 \mathrm{~g} . \mathrm{g}^{-1}$ on sorbitol compared to 0.41 and $0.44 \mathrm{~g}^{\mathrm{g}} \mathrm{g}^{-1}$ on glucose, correspondingly, assuming the same sugar transport mechanism) and higher $\mathrm{CO}_{2}$ availability, favouring carbon flux through the $\mathrm{C} 4$ branch, as 
a consequence of higher reducing power and higher PPCK carboxylation activity. Moreover, flexibility in sugar transport mechanisms or enzymes reversibility (e.g. ME2) may lead to increased levels of SA, as carbon flux partitioning between $\mathrm{C} 3$ and $\mathrm{C} 4$ pathways was shown to be largely affected (Fig. 4). The reverse activity of ME2 has shown to increase SA yields, redirecting part of the carbon flux from the $\mathrm{C} 3$ toward the $\mathrm{C} 4$ branch through the carboxylation of pyruvate to L-malate, with the simultaneous production of reducing power (NADPH). On the other hand, transport activities limited to PEP:PTS-based systems decrease SA yields, since PEP is used as the energy source for sugar uptake generating pyruvate, which is necessarily consumed via the $\mathrm{C} 3$ branch.

\section{Conclusions}

In this work, the GSMM of A. succinogenes $130 \mathrm{Z}$ ( $i \mathrm{BP} 722$ ) was reconstructed and validated using different sets of experimental data from literature. The reconstruction of the model included the compilation of functionally annotated metabolic genes and the corresponding coding proteins, as well as associated biochemical reactions. The model was complemented with a biomass equation and spontaneous and transport reactions. It was further amended after a gap filling process, including the identification of genetic evidences based on homology searches. Model accuracy to predict growth phenotypes and the production of fermentative by-products was evaluated using FVA and pFBA simulation methods. The ability to predict changes in carbon flux distributions due to environmental perturbations like $\mathrm{CO}_{2}$ limitations or alterations in the redox state was also tested. Predicted SA yields were in good agreement with experimental data, suggesting that the model is able to characterize the fermentative metabolism under various conditions. The increase in $\mathrm{CO}_{2}$ availability showed to have a positive impact in SA yields, which is consistent with reported data $[48,49]$. As such, optimal conditions for increased SA yields may include increased $\mathrm{CO}_{2}$ availability, the use of more reduced carbon sources like sorbitol or the use of external energy source like $\mathrm{H}_{2}$ [33]. Besides improving process conditions for the production of SA, the design of microbial strains by metabolic engineering to increase the flux through the $\mathrm{C} 4$ branch has been attempted, albeit with limited success [70]. Model predictions show that changes in sugar transport systems, $\mathrm{CO}_{2}$ fixation activities or the reversibility of the malic enzyme may have an impact in the SA yield. Therefore, it is expected that modifications in the metabolism that would include these activities would further improve the SA yield.

Overall, the $i$ BP722 model enables a better understanding of the metabolic behaviour and capabilities of this organism, which can be explored to further improve SA productivity. The capacity to consume a wide range of $\mathrm{C} 5$ and C6 sugars, as well as other low-cost carbon sources (e.g. glycerol or lactose) and its metabolic flexibility may provide some advantages over other SA-producing strains, like recombinant Escherichia coli or Mannheimia succiniciproducens [69].

\section{Additional files}

Additional file 1: Biomass composition of A. succinogenes $130 Z$. (DOCX $43 \mathrm{~kb}$ )

Additional file 2: Details on the iBP722 model. (DOCX 24 kb) Additional file 3: Testing $A$. succinogenes growth on different carbon sources. (DOCX $22 \mathrm{~kb}$ )

Additional file 4: Details on transport mechanisms included in the iBP722 model. (DOCX 37 kb)

Additional file 5: Growth predictions on glycerol with and without DMSO. (DOCX 21 kb)

\section{Abbreviations}

Metabolites

AA: Acetic acid; AA ex: Extracellular acetic acid; ACCOA: Acetyl-coenzyme A; E4P: Erythrose-4-phosphate; EtOH: Ethanol; EtOH $\mathrm{ex}_{\text {: }}$ Extracellular ethanol; F6P: Fructose-6-phosphate; FA: Formic acid; FA ex: Extracellular formic acid; FUM: Fumarate; FUM ex: Extracellular fumarate; G3P: Glyceraldehyde-3phosphate; G6P: Glucose-6-phosphate; Glcex: Extracellular glucose; MAL: Malate; OAA: Oxaloacetate; PEP: Phosphoenolpyruvate; PYR: Pyruvate; R5P: Ribose-5-phosphate; S7P: Sedoheptulose-7-phosphate; SA: Succinic acid. $S A_{e x}$ : Extracellular succinic acid

\section{Reactions}

ACKr: Acetate kinase; ALCD2x: Alcohol dehydrogenase; ENO: Enolase; EX_CO $\mathrm{CO}_{2}$ : Carbon dioxide exchange reaction; EX_AA: Acetic acid exchange reaction; EX_EtOH: Ethanol exchange reaction; EX_FA: Formic acid exchange reaction; EX_FUM: Fumarate exchange reaction; EX_Glc: Glucose exchange reaction; EX_SA: Succinic acid exchange reaction; FDHmq: Formate dehydrogenase; FRD2: Fumarate reductase; FUM: Fumarase; G6PDH2r: Glucose 6-phosphate dehydrogenase of the oxidative pentose phosphate pathway; ME2: Malic enzyme; OAADC: Oxaloacetate decarboxylase; PFK: Phosphofructokinase; PFL: Pyruvate formate-lyase; PGI: Glucose-6-phosphate isomerase; PPCK: PEP carboxykinase; PYK: Pyruvate kinase

\section{Acknowledgements}

The authors want to thank Apostolis Koutinas and co-workers from Agricultural University of Athens for the helpful discussions.

\section{Funding}

Financially supported by BRIGIT (KBBE-2012-6-311935, FP7 project Contract nr 311935) and by the Portuguese Foundation for Science and Technology (FCT) under the scope of the strategic funding of UID/BIO/04469/2013 unit and COMPETE 2020 (POCI-01-0145-FEDER-006684), in addition to the BioTecNorte operation (NORTE-01-0145-FEDER-000004) funded by European Regional Development Fund under the scope of Norte2020 - Programa Operacional Regional do Norte.

\section{Availability of data and materials}

The GSMM generated during the current study is available at http:// darwin.di.uminho.pt/models and at BioModels with the identifier MODEL1804130001. A. succinogenes $130 Z$ complete genome - GenBank accession number NC_009655.

\section{Authors' contributions}

Model development: SC, BP, JM, PV; Design of simulation scenarios: PV, SS, IR: Model simulations and data analysis SC, BP, PV; Paper writing: SC, BP; Contribution to paper writing: PV, SS, IR. All authors read and approved the final version of the manuscript. 


\section{Ethics approval and consent to participate}

Not applicable.

\section{Competing interests}

The authors declare that they have no competing interests

\section{Publisher's Note}

Springer Nature remains neutral with regard to jurisdictional claims in published maps and institutional affiliations.

\section{Author details}

${ }^{1}$ SilicoLife Lda, Rua do Canastreiro 15, 4715-387 Braga, Portugal. ${ }^{2}$ CEB Centre of Biological Engineering, University of Minho, Campus de Gualtar, 4710-057 Braga, Portugal. ${ }^{3}$ Instituto de Tecnologia Química e Biológica António Xavier, Universidade Nova de Lisboa (ITQB-NOVA), Oeiras, Portugal.

\section{Received: 21 December 2017 Accepted: 14 May 2018}

\section{Published online: 30 May 2018}

\section{References}

1. Van der Werf MJ, Guettler MV, Jain MK, Zeikus JG. Environmental and physiological factors affecting the succinate product ratio during carbohydrate fermentation by Actinobacillus sp. 130Z. Arch Microbiol. 1997; 167:332-42.

2. Liu YP, Zheng P, Sun ZH, Ni Y, Dong JJ, Zhu LL. Economical succinic acid production from cane molasses by Actinobacillus succinogenes. Bioresour Technol. 2008;99:1736-42.

3. Urbance SE, Pometto AL, DiSpirito A a., Denli Y: Evaluation of succinic acid continuous and repeat-batch biofilm fermentation by Actinobacillus succinogenes using plastic composite support bioreactors. Appl Microbiol Biotechnol 2004, 65:664-670.

4. Guettler MV, Rumler D, Jainf MK. Actinobacillus succinogenes sp. nov., a novel succinic-acid-producing strain from the bovine rumen. Int J Syst Bacteriol. 1999;49(1 999):207-16.

5. Gunnarsson IB, Alvarado-morales $\mathrm{M}$, Angelidaki I. Utilization of $\mathrm{CO} 2$ fixating bacterium Actinobacillus succinogenes $130 Z$ for simultaneous biogas upgrading and biosuccinic acid production. Environ Sci Technol. 2014;48: 12464-8.

6. Shen N, Qin Y, Wang Q, Liao S, Zhu J, Zhu Q, Mi H, Adhikari B, Wei Y, Huang R. Production of succinic acid from sugarcane molasses supplemented with a mixture of corn steep liquor powder and peanut meal as nitrogen sources by Actinobacillus succinogenes. Lett Appl Microbiol. 2015;60:544-51.

7. Z Zheng P, Dong JJ, Sun ZH, Ni Y, Fang L. Fermentative production of succinic acid from straw hydrolysate by Actinobacillus succinogenes. Bioresour Technol. 2009;100:2425-9.

8. Wan C, Li Y, Shahbazi A, Xiu S. Succinic acid production from cheese whey using Actinobacillus succinogenes 130 Z. Appl Biochem Biotechnol. 2008;145: $111-9$.

9. Chen $K$, Jiang M, Wei P, Yao J, Wu H. Succinic acid production from acid hydrolysate of corn fiber by Actinobacillus succinogenes. Appl Biochem Biotechnol. 2010;160:477-85.

10. Jiang $M$, Chen $K$, Liu Z, Wei $P$, Ying $H$, Chang $H$. Succinic acid production by Actinobacillus succinogenes using spent brewer's yeast hydrolysate as a nitrogen source. Appl Biochem Biotechnol. 2010;160:244-54.

11. Xi YL, Chen KQ, Dai WY, Ma JF, Zhang M, Jiang M, Wei P, Ouyang PK. Succinic acid production by Actinobacillus succinogenes NJ113 using corn steep liquor powder as nitrogen source. Bioresour Technol. 2013;136:775-9.

12. Li Q, Yang M, Wang D, Li W, Wu Y, Zhang Y, Xing J, Su Z. Efficient conversion of crop stalk wastes into succinic acid production by Actinobacillus succinogenes. Bioresour Technol. 2010;101:3292-4.

13. Chen $\mathrm{K}$, Zhang $\mathrm{H}$, Miao $\mathrm{Y}$, Jiang $M$, Chen J. Succinic acid production from enzymatic hydrolysate of sake lees using Actinobacillus succinogenes $130 Z$. Enzym Microb Technol. 2010;47:236-40.

14. Chen KQ, Li J, Ma JF, Jiang M, Wei P, Liu ZM, Ying HJ. Succinic acid production by Actinobacillus succinogenes using hydrolysates of spent yeast cells and corn fiber. Bioresour Technol. 2011;102:1704-8.

15. Yu J, Li Z, Ye Q, Yang Y, Chen S. Development of succinic acid production from corncob hydrolysate by Actinobacillus succinogenes. J Ind Microbiol Biotechnol. 2010;37:1033-40.
16. Zeikus JG, Jain MK, Elankovan P. Biotechnology of succinic acid production and markets for derived industrial products. Appl Microbiol Biotechnol. 1999;51:545-52.

17. Kamzolova SV, Vinokurova NG, Shemshura ON, Bekmakhanova NE, Lunina JN, Samoilenko VA, Morgunov IG. The production of succinic acid by yeast Yarrowia lipolytica through a two-step process. Appl Microbiol Biotechnol. 2014;98:7959-69.

18. Raab AM, Gebhardt G, Bolotina N, Weuster-Botz D, Lang C. Metabolic engineering of Saccharomyces cerevisiae for the biotechnological production of succinic acid. Metab Eng. 2010;12:518-25.

19. Yan D, Wang C, Zhou J, Liu Y, Yang M, Xing J. Construction of reductive pathway in Saccharomyces cerevisiae for effective succinic acid fermentation at low pH value. Bioresour Technol. 2014;156:232-9.

20. Liu Y, Wu H, Li Q, Tang X, Li Z, Ye Q. Process development of succinic acid production by Escherichia coli NZN111 using acetate as an aerobic carbon source. Enzym Microb Technol. 2011;49:459-64.

21. Scholten E, Renz T, Thomas J. Continuous cultivation approach for fermentative succinic acid production from crude glycerol by Basfia succiniciproducens DD1. Biotechnol Lett. 2009;31:1947-51.

22. Kim DY, Yim SC, Lee PC, Lee WG, Lee SY, Chang HN. Batch and continuous fermentation of succinic acid from wood hydrolysate by Mannheimia succiniciproducens MBEL55E. In Enzyme Microb Technol Volume. 2004;35:648-53.

23. Lee SY, Kim JM, Song H, Lee JW, Kim TY, Jang Y-S. From genome sequence to integrated bioprocess for succinic acid production by Mannheimia succiniciproducens. Appl Microbiol Biotechnol. 2008;79:11-22.

24. Myriant [http://www.myriant.com].

25. BASF [http://www.basf.com].

26. BioAmber [http://www.bio-amber.com]

27. Cok B, Tsiropoulos I, Roes AL, Patel MK. Succinic acid production derived from carbohydrates: an energy and greenhouse gas assessment of a platform chemical toward a bio-based economy. Biofuels Bioprod Biorefin. 2014;8:16-29.

28. Choi S, Song CW, Shin JH, Lee SY. Biorefineries for the production of top building block chemicals and their derivatives. Metab Eng. 2015:223-39.

29. Jansen MLA, van Gulik WM. Towards large scale fermentative production of succinic acid. Curr Opin Biotechnol. 2014;30:190-7.

30. Lee SJ, Song H, Lee SY. Genome-based metabolic engineering of Mannheimia succiniciproducens for succinic acid production. Appl Environ Microbiol. 2006;72:1939-48.

31. Yan $Q$, Zheng $P$, Dong JJ, Sun ZH. A fibrous bed bioreactor to improve the productivity of succinic acid by Actinobacillus succinogenes. J Chem Technol Biotechnol. 2014;89:1760-6.

32. McKinlay JB, Zeikus JG, Vieille C. Insights into Actinobacillus succinogenes fermentative metabolism in a chemically defined growth medium. Appl Environ Microbiol. 2005;71:6651-6.

33. McKinlay JB, Vieille C. 13C-metabolic flux analysis of Actinobacillus succinogenes fermentative metabolism at different $\mathrm{NaHCO} 3$ and $\mathrm{H}_{2}$ concentrations. Metab Eng. 2008;10:55-68.

34. Li J, Jiang M, Chen K-QQ, Ye Q, Shang L-AA, Wei P, Ying H-JJ, Chang H-NN. Effect of redox potential regulation on succinic acid production by Actinobacillus succinogenes. Bioprocess Biosyst Eng. 2010;33:911-20.

35. Carvalho M, Matos M, Roca C, Reis M a M. Succinic acid production from glycerol by Actinobacillus succinogenes using dimethylsulfoxide as electron acceptor. New Biotechnol. 2014;31:133-9.

36. van Heerden CD, Nicol W. Continuous succinic acid fermentation by Actinobacillus succinogenes. Biochem Eng J. 2013;73:5-11.

37. Otero JM, Cimini D, Patil KR, Poulsen SG, Olsson L, Nielsen J. Industrial systems biology of Saccharomyces cerevisiae enables novel succinic acid cell factory. PLoS One. 2013;8

38. Sajo Mienda B, Shahir Shamsir M. Model-driven in silico glpC gene knockout predicts increased succinate production from glycerol in Escherichia coli. AIMS Bioeng. 2015;2:40-8.

39. Xu G, Zou W, Chen X, Xu N, Liu L, Chen J. Fumaric acid production in Saccharomyces cerevisiae by in silico aided metabolic engineering. PLoS One. 2012;7

40. Raab AM, Lang C. Oxidative versus reductive succinic acid production in the yeast Saccharomyces cerevisiae. Bioeng Bugs. 2011;2

41. Cheng KK, Wang GY, Zeng J, Zhang JA. Improved succinate production by metabolic engineering. Biomed Res Int. 2013;2013

42. Lin H, Bennett GN, San KY. Fed-batch culture of a metabolically engineered Escherichia coli strain designed for high-level succinate production and yield under aerobic conditions. Biotechnol Bioeng. 2005;90:775-9. 
43. Liu R, Liang L, Chen K, Ma J, Jiang M, Wei P, Ouyang P. Fermentation of xylose to succinate by enhancement of ATP supply in metabolically engineered Escherichia coli. Appl Microbiol Biotechnol. 2012;94:959-68.

44. Zhang X, Jantama K, Moore JC, Jarboe LR, Shanmugam KT, Ingram LO Metabolic evolution of energy-conserving pathways for succinate production in Escherichia coli. Proc Natl Acad Sci U S A. 2009;106:20180-5.

45. ya $L L$, ming $L R$, feng $M J$, quan $C K$, Jiang $M$, Wei $P$. Increased production of succinic acid in Escherichia coli by overexpression of malate dehydrogenase. Biotechnol Lett. 2011;33:2439-44.

46. Yang J, Wang Z, Zhu N, Wang B, Chen T, Zhao X. Metabolic engineering of Escherichia coli and in silico comparing of carboxylation pathways for high succinate productivity under aerobic conditions. Microbiol Res. 2014;169: 432-40.

47. Jiang M, Ma J, Wu M, Liu R, Liang L, Xin F, Zhang W, Jia H, Dong W. Progress of succinic acid production from renewable resources: metabolic and fermentative strategies. Bioresour Technol. 2017;

48. Zou W, Zhu L-W, Li H-M, Tang Y-J. Significance of $\mathrm{CO} 2$ donor on the production of succinic acid by Actinobacillus succinogenes ATCC 55618. Microb Cell Factories. 2011;10:87.

49. Xi Y, Chen K, Li J, Fang X, Zheng X, Sui S, Jiang M, Wei P. Optimization of culture conditions in $\mathrm{CO} 2$ fixation for succinic acid production using Actinobacillus succinogenes. J Ind Microbiol Biotechnol. 2011;38:1605-12.

50. McKinlay JB, Laivenieks M, Schindler BD, McKinlay A a, Siddaramappa S, Challacombe JF, Lowry SR, Clum A, Lapidus AL, Burkhart KB, Harkins V, Vieille C. A genomic perspective on the potential of Actinobacillus succinogenes for industrial succinate production. BMC Genomics 2010, 11:680.

51. McKinlay JB, Shachar-Hill Y, Zeikus JG, Vieille C. Determining Actinobacillus succinogenes metabolic pathways and fluxes by NMR and GC-MS analyses of 13C-labeled metabolic product isotopomers. Metab Eng. 2007;9:177-92.

52. Altschul SF, Gish W, Miller W, Myers EW, Lipman DJ. Basic local alignment search tool. J Mol Biol. 1990;215(3):403-10.

53. Eddy SR. Profile hidden Markov models. Bioinformatics. 1998;14(9):755-63.

54. The UniProt Consortium. UniProt: the universal protein knowledgebase. Nucleic Acids Res. 2018;46(5):2699.

55. Dias O, Rocha M, Ferreira EC, Rocha I. Reconstructing genome-scale metabolic models with merlin. Nucleic Acids Research. 2015;43(8):3899-910.

56. Kanehisa M, Goto S. KEGG: Kyoto Encyclopaedia of genes and genomes. Nucl Acids Res. 2000;28:27-30.

57. Saier MH, Reddy VS, Tamang DG, Västermark Å. The transporter classification database. Nucleic Acids Res. 2014:42

58. Caspi R, Billington R, Ferrer L, Foerster H, Fulcher CA, Keseler IM, Kothari A, Krummenacker M, Latendresse M, Mueller LA, Ong $Q$, Paley S, Subhraveti $P$, Weaver DS, Karp PD. The MetaCyc database of metabolic pathways and enzymes and the BioCyc collection of pathway/genome databases. Nucleic Acids Res. 2016;44:D471-80.

59. Flamholz A, Noor E, Bar-even A, Milo R. EQuilibrator - the biochemical thermodynamics calculator. Nucleic Acids Res. 2012;40

60. Santos $\mathrm{S}$, Rocha I. A computation tool for the estimation of biomass composition from genomic and transcriptomic information. Adv. Intell. Syst. Comput. 2016:161-9.

61. Juty N, Ali R, Glont M, Keating S, Rodriguez N, Swat MJ, Wimalaratne SM Hermjakob H, Le Novère N, Laibe C, Chelliah V. BioModels: content, features, functionality, and use. CPT Pharmacometrics Syst Pharmacol. 2015:55-68.

62. Rocha I, Maia P, Evangelista P, Vilaça P, Soares S, Pinto JP, Nielsen J, Patil KR, Ferreira EC, Rocha M. OptFlux: an open-source software platform for in silico metabolic engineering. BMC Syst Biol. 2010;4:45.

63. Bradfield MFA, Nicol W. Continuous succinic acid production from xylose by Actinobacillus succinogenes. Bioprocess Biosyst Eng. 2016;39:233-44.

64. Li J, Jiang M, Chen K, Shang L, Wei P, Ying H, Ye Q, Ouyang P, Chang H. Enhanced production of succinic acid by Actinobacillus succinogenes with reductive carbon source. Process Biochem. 2010;45:980-5.

65. Schindler BD, Joshi RV, Vieille C. Respiratory glycerol metabolism of Actinobacillus succinogenes $130 Z$ for succinate production. J Ind Microbiol Biotechnol. 2014;41:1339-52.

66. Lin SKC, Du C, Koutinas A, Wang R, Webb C. Substrate and product inhibition kinetics in succinic acid production by Actinobacillus succinogenes. Biochem Eng J. 2008:41:128-35.

67. Orth JD, Conrad TM, Na J, Lerman JA, Nam H, Feist AM, Palsson BØ. A comprehensive genome-scale reconstruction of Escherichia coli metabolism—2011. Mol Syst Biol. 2011;
68. Mo ML, Palsson BO, Herrgård MJ. Connecting extracellular metabolomic measurements to intracellular flux states in yeast. BMC Syst Biol. 2009;3:37.

69. Pateraki C, Patsalou M, Vlysidis A, Kopsahelis N, Webb C, Koutinas AA, Koutinas M. Actinobacillus succinogenes: advances on succinic acid production and prospects for development of integrated biorefineries. Biochem Eng J. 2016;112:285-303.

70. Guarnieri MT, Chou YC, Salvachúa D, Mohagheghi A, St. John PC, Peterson DJ, Bomble YJ, Beckham GT. Metabolic engineering of Actinobacillus succinogenes provides insights into succinic acid biosynthesis. Appl Environ Microbiol. 2017;83

71. Song H, Lee JW, Choi S, You JK, Hong WH, Lee SY. Effects of dissolved CO2 levels on the growth of Mannheimia succiniciproducens and succinic acid production. Biotechnol Bioeng. 2007:98:1296-304.

72. Brink HG, Nicol W. Succinic acid production with Actinobacillus succinogenes: rate and yield analysis of chemostat and biofilm cultures. Microb Cell Factories. 2014;13:111.

73. Lin H, Bennett GN, San KY. Metabolic engineering of aerobic succinate production systems in Escherichia coli to improve process productivity and achieve the maximum theoretical succinate yield. Metab Eng. 2005;7:116-27.

\section{Ready to submit your research? Choose BMC and benefit from:}

- fast, convenient online submission

- thorough peer review by experienced researchers in your field

- rapid publication on acceptance

- support for research data, including large and complex data types

- gold Open Access which fosters wider collaboration and increased citations

- maximum visibility for your research: over $100 \mathrm{M}$ website views per year

At BMC, research is always in progress.

Learn more biomedcentral.com/submissions 